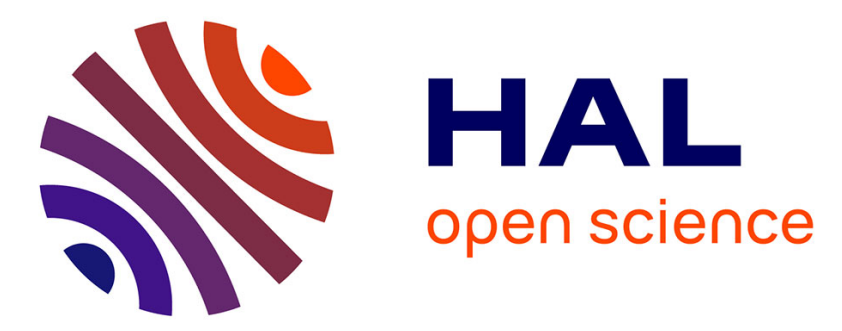

\title{
Using Temporal Logic for Dynamic Reconfigurations of Components
}

\author{
Julien Dormoy, Olga Kouchnarenko, Arnaud Lanoix
}

\section{To cite this version:}

Julien Dormoy, Olga Kouchnarenko, Arnaud Lanoix. Using Temporal Logic for Dynamic Reconfigurations of Components. 7th International Workshop on Formal Aspects of Component Software FACS'2010, Oct 2010, Guimaraes, Portugal. inria-00541613

\section{HAL Id: inria-00541613 https://hal.inria.fr/inria-00541613}

Submitted on 30 Nov 2010

HAL is a multi-disciplinary open access archive for the deposit and dissemination of scientific research documents, whether they are published or not. The documents may come from teaching and research institutions in France or abroad, or from public or private research centers.
L'archive ouverte pluridisciplinaire HAL, est destinée au dépôt et à la diffusion de documents scientifiques de niveau recherche, publiés ou non, émanant des établissements d'enseignement et de recherche français ou étrangers, des laboratoires publics ou privés. 


\title{
Using Temporal Logic for Dynamic Reconfigurations of Components
}

\author{
Julien Dormoy ${ }^{1}$, Olga Kouchnarenko ${ }^{1}$, and Arnaud Lanoix ${ }^{2}$ \\ 1 University of Franche-Comté, Besançon, France \\ \{jdormoy, okouchnarenko\}@lifc.univ-fcomte.fr \\ 2 Nantes University, Nantes, France \\ arnaud.lanoix@univ-nantes.fr
}

\begin{abstract}
Dynamic reconfigurations increase the availability and the reliability of component-based systems by allowing their architectures to evolve at run-time. This paper deals with the formal specification and verification of dynamic reconfigurations of those systems using architectural constraints and temporal logic patterns.

The proposals of the paper are applied to the Fractal component model. Given a Fractal reference implementation of a component-based system, we specify its dynamic reconfigurations using a temporal pattern logic for Fractal, called FTPL, characterizing the correct behaviour of the system under some architectural constraints. We study system reconfigurations on which we verify these requirements, in particular by reusing the FPath and FScript tools.
\end{abstract}

\section{Introduction}

Component-based development provides significant advantages like portability, adaptability, re-usability, etc. when developing, e.g., Java Card smart card applications or when composing components or services within Service Component Architecture (SCA). The adaptability means that component-based systems must be adapted, or even adapt themselves [15] to their environments during their lifetime, and there is a need to support dynamic reconfigurations, including unanticipated ones [20]. To take up this challenge, this paper deals with the formal specification and verification of dynamic reconfigurations of componentbased systems, and uses temporal patterns to monitor them.

The present paper makes the following contributions. The first contribution is a formal definition of the semantics of component-based systems with reconfigurations. To specify system reconfigurations, the second contribution is the definition of a linear time temporal logic based on architectural constraints which are first order configuration properties, and on event properties. For temporal operators, its expressive power is related to the well known linear time temporal logic (LTL) [19]. The third contribution is the application of the paper proposals to the Fractal component model [10]. For the Fractal component model, run-time verification issues are addressed to monitor reconfigurations during system lifetime. 
More precisely, this paper follows the line of reasoning suggested in [12], where the system consistency during its dynamic reconfigurations relies on integrity constraints - predicates on assemblies of architectural elements and component states. To go further, we propose to support dynamic reconfigurations by using more complex architectural constraints and linear temporal logic patterns. These temporal patterns have been inspired by the pragmatic work of the SanTos Specification Pattern Project [13], and works on temporal extension of JML [21, $9,14]$ helping Java programmers in writing formal specifications. We refer to this temporal extension as FTPL, for Temporal Pattern Language, prefixed by an ' $\mathrm{F}$ ' to denote its adaptation to Fractal-like component systems and to first-order integrity constraints over them.

The proposals of the paper are applied to the Fractal component model. Given a Fractal reference implementation of a component-based system, we specify its dynamic reconfigurations using FTPL, characterizing the correct behaviour of the system under some architectural constraints. We monitor system reconfigurations by reusing the FPath and FScript tool supports.

The remainder of the paper is organised as follows. After giving a motivating example in Sect. 2, we formally define the semantics of component-based systems with reconfigurations in Sect. 3. To support system reconfigurations, Sect. 4 introduces a linear time temporal logic based on architectural constraints and events. Then, the proposals of the paper are applied to and illustrated on the Fractal component model in Sect. 5. Finally, Section 6 concludes before discussing related work.

\section{Motivating Example}

To motivate and to illustrate our approach, let us consider an example of a HTTP server from [11]. The architecture of this server is displayed in Fig. 1.

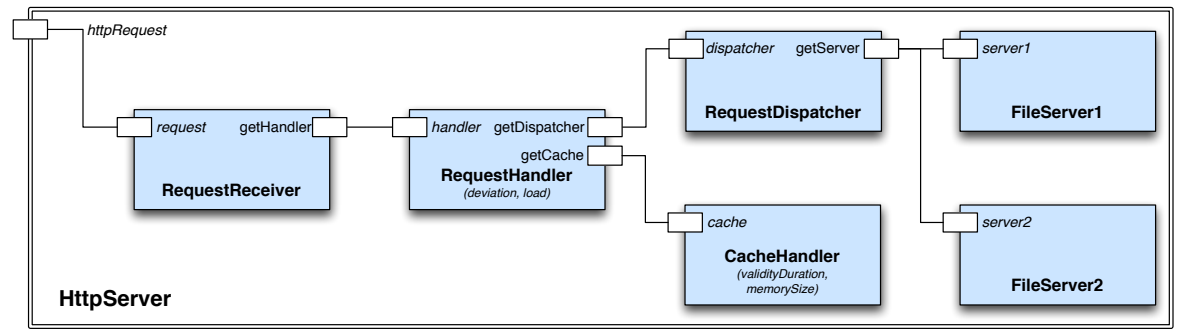

Fig. 1. HTTP Server architecture

The RequestReceiver component reads HTTP requests from the network and transmits them to the RequestHandler component. In order to perform a request, RequestHandler can either use the cache (with the component 
CacheHandler) or transmit the request to the RequestDispatcher component. This component uses a set of file servers (like the FileServer1 and FileServer2 components) to answer the request.

This architecture provides a cache (CacheHandler component) and a load controller (RequestDispatcher component) in order to control the response time of the HTTP server. To keep the response time as short as possible whatever the number of requests is, in [11] the authors propose to dynamically reconfigure the HTTP server. For that, some requirements have been identified:

1. The CacheHandler component is used only if the number of similar HTTP requests is high.

2. The quantity of allocated memory for the CacheHandler component must depend on the overall load of the server.

3. The validity of data in the cache must also depend on the overall load of the server.

4. The number of used file servers depends on the overall load of the server.

In order to take these requirements into account, the RequestHandler and CacheHandler components are equipped with some parameters. The number of requests (load) and the percentage of similar requests (deviation) are two parameters defined for the RequestHandler component. The memory size (memorySize) and the data validity duration (validityDuration) are two parameters defined for the CacheHandler component.

We consider that the HTTP server can be reconfigured during the execution by the following reconfigurations

1. AddCacheHandler and RemoveCacheHandler which are respectively used to add and remove the CacheHandler component,

2. AddFileServer and removeFileServer which are respectively used to add and remove the FileServer2 component,

3. MemorySizeUp and MemorySizeDown which are respectively used to increase and to decrease the MemorySize value,

4. DurationValidityUp and DurationValidityDown to respectively increase and decrease the ValidityDuration value.

\section{Architectural (Re-)Configuration Model}

This section gives means for specifying component-based systems with reconfigurations. A model we propose here is inspired by the model in $[16,18]$ given for Fractal. Both models are graphs allowing one to represent component-based architectures and reconfiguration operations and to reason about them. Unlike $[16,18]$, in our model, only the basic and generic concepts are considered to allow their application to various hierarchical component models: components as runtime entities, required and provided interfaces as interaction points between components, bindings to link component interfaces. Components are either primitive components or composite components, and primitive components can have some attributes used as configuration parameters. 
Basically, a component-based system with reconfigurations is characterized by a set of configurations and a set of actions that allow the modification of configurations.

\subsection{Component-based architectures}

In general, the system configuration is the specific definition of the elements that define or prescribe what a system is composed of. We define a configuration to be a set of architectural elements (components, interfaces and parameters) together with a relation to structure and to link them. We consider a graphbased representation as proposed in $[16,18]$.

Definition 1. A configuration $c$ is a tuple $\langle$ Elem, Rel $\rangle$ where:

- Elem is a set of architectural elements, and

- Rel $\subseteq$ Elem $\times$ Elem is a relation between architectural elements.

In our model, the architectural elements are the core entities of a componentbased system: components, required or provided interfaces, and parameters.

Definition 2. The set of architectural elements Elem is defined by:

$$
\text { Elem }=\text { Component } \uplus \text { Interface } \uplus \text { Parameter } \uplus \text { Type }
$$

where

- Component is a non-empty set of the core entities, i.e components;

- Interface $=$ Required $\uplus$ Provided is a set of the (required and provided) interfaces;

- Parameter is a set of component parameters;

- Type is a set of data types associated with parameters.

Each data type is a set of data values. For the sake of readability, we identify data type names with the corresponding data domains.

The architectural relation $R e l$ then expresses various links between architectural elements. For example, it allows specifying that a component has an interface or a parameter, or that a component contains other (sub-)components, or that an interface is linked to another one.

Definition 3. The architectural relation Rel is defined by:

$$
R e l=\begin{aligned}
& \text { ProvidedBy } \uplus \text { RequiredBy } \uplus \text { ParameterOf } \uplus \\
& \text { Type } O f \uplus \text { ValueOf } \uplus \text { ChildOf } \uplus \text { Binding } \uplus \text { Delegate }
\end{aligned}
$$

where

- ProvidedBy : Provided $\rightarrow$ Component is a total surjective function which gives the component having a provided interface;

- RequiredBy : Required $\rightarrow$ Component is a total function which gives the component with a required interface; 
- ParameterOf : Parameter $\rightarrow$ Component is a total function which gives the component of a considered parameter;

- TypeOf : Parameter $\rightarrow$ Type is a total function which gives the type associated with a considered parameter;

- ValueOf : Parameter $\rightarrow \bigcup_{\text {type } \in \text { Type }}$ type such that $\forall p \in$ Parameter : $\operatorname{ValueO} f(p) \in \operatorname{TypeO} f(p)$, is a total function which gives the current value of a considered parameter;

- ChildOf $\subseteq$ Component $\times$ Component is a irreflexive and antisymetric relation linking composite components to their sub-components ${ }^{3}$ such that:

- $\forall c, c^{\prime} \in$ Component. $\left(\left(c, c^{\prime}\right) \in\right.$ ChildOf $\Rightarrow \forall p \in$ Parameter. (ParameterOf $(p) \neq c)$ ), i.e, composite components have no parameter;

- Let ChildOf ${ }^{+}$be the transitive closure of ChildOf. Then, $\forall c, c^{\prime} \in$ Component. $\left(\left(c, c^{\prime}\right) \in C\right.$ hild $\left.O f^{+} \Rightarrow c \neq c^{\prime}\right)$, i.e., ChildOf is an acyclic relation;

- Binding : Provided $\rightarrow$ Required is a partial function such that $\forall i p \in$ Provided, ir $\in$ Required. (Binding $(i p)=i r \Rightarrow$ ProvidedBy(ip) $\neq$ RequiredBy(ir $) \wedge \exists c \in$ Component. $((c$, ProvidedBy $(i p)) \in$ ChildOf $\wedge$ $(c$, RequiredBy $(i r)) \in C$ Child $O f)$ ), i.e., two linked interfaces do not belong to the same component, but the corresponding components are sub-components of the same composite component;

- Delegate : Interface $\rightarrow$ Interface is a partial injective function to specify the delegation from a sub-component interface to the composite interface such that $\forall i, i^{\prime} \in$ Interface. $\left(\right.$ Delegate $(i)=i^{\prime} \Rightarrow\left(\right.$ ProvidedBy $\left(i^{\prime}\right)$, ProvidedBy $\left.(i)\right) \in$ ChildOf $\vee\left(\right.$ RequiredBy $\left(i^{\prime}\right)$, RequiredBy $\left.(i)\right) \in$ ChildOf $)$, i.e., when delegating, the component providing $i$ is a sub-component of the component providing $i^{\prime}$, or the component requiring $i$ is a sub-component of the component requiring $i^{\prime}$.

Example 1. Figure 2 illustrates main lines of Definition 3 on the example from Section 2. In this figure, the architectural elements are depicted as boxes and circles, whereas architectural relations are represented by arrows. For example, the request architectural element (at the bottom on the left) is an interface provided by RequestReceiver. The RequestReceiver architectural element is a subcomponent of the HttpServer composite component which provides the httpRequest interface. The request interface delegates results passing to the httpRequest interface.

\subsection{Dynamicity of Component Architectures}

To support system evolution, some component models provide mechanisms to dynamically reconfigure the component-based architecture, during their execution. These dynamic reconfigurations are then based on architectural modifications, among the following primitive operations:

\footnotetext{
${ }^{3}$ For any $(p, q) \in$ Child $O f$, we say that $p$ has a sub-component $q$, i.e. $q$ is a child of $p$.
} 


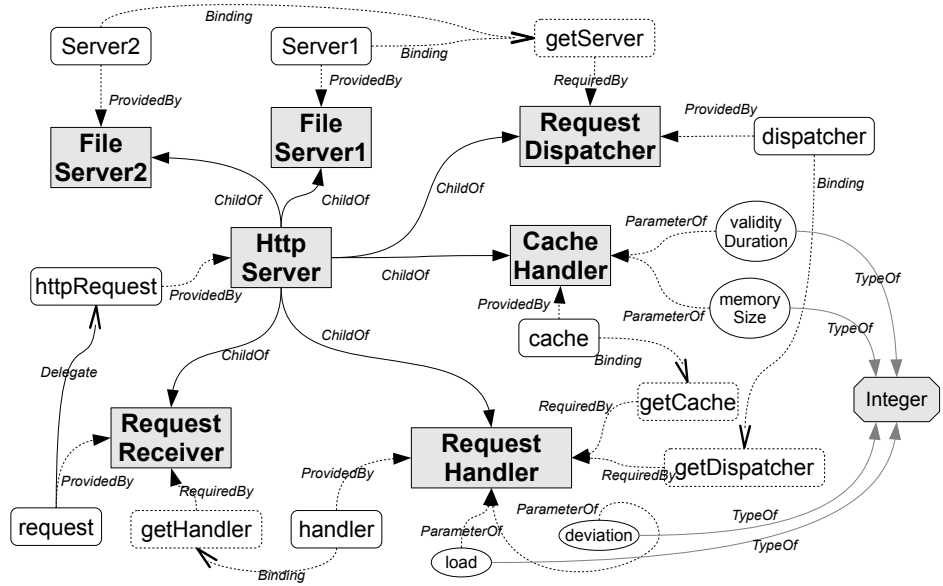

Fig. 2. Graph representation of the HTTP Server example

- instantiation/destruction of components;

- addition/removal of components;

- binding/unbinding of component interfaces;

- starting/stopping components;

- setting parameter values of components;

or combinations of them. Notice that reconfigurations are not the only manner to make a component architecture evolve. The normal running of different components also changes the architecture by modifying parameter values or stopping components, like in the example.

Considering the component-based architecture model given in Sect. 3.1, an operation which makes the component architecture evolve by a reconfiguration action or by running, is modelled by a graph transformation operation adding or removing nodes and/or arcs in the graph of the configuration. An evolution operation op transforms a configuration $c=\langle$ Elem, Rel $\rangle$ into another one $c^{\prime}=\left\langle E l e m^{\prime}, R e l^{\prime}\right\rangle$. It is represented by a transition from $c$ to $c^{\prime}$, noticed $c \stackrel{o p}{\rightarrow} c^{\prime}$. Among the evolution operations (running operations and reconfigurations), we particularly focus on the reconfiguration ones, which are either the above-mentioned primitive architectural operations or their compositions. The remaining running operations are all represented by a generic operation, called the run operation; it is also the case for sequences of running operations.

Definition 4. The set of evolution operations $\mathcal{R}_{\text {run }}$ is defined by:

$$
\mathcal{R}_{\text {run }}=\mathcal{R} \cup\{\text { run }\}
$$

with

$-\mathcal{R}$ is a finite set of reconfiguration operations; 
- run is an action renaming one or more running operations.

Given a component architecture and the set $\mathcal{R}_{\text {run }}$ of reconfiguration operations, the behaviour of the component architecture is defined as a transition system labelled over $\mathcal{R}_{\text {run }}$.

Definition 5. The evolution of a component architecture is defined by the transition system $\left\langle\mathcal{C}, \mathcal{R}_{\text {run }}, \rightarrow\right\rangle$ where:

$-\mathcal{C}=\left\{c, c_{1}, c_{2}, \ldots\right\}$ is a set of configurations,

- $\mathcal{R}_{\text {run }}$ is a finite set of evolution operations,

$-\rightarrow \subseteq \mathcal{C} \times \mathcal{R}_{\text {run }} \times \mathcal{C}$ is the reconfiguration relation.

Given the evolution of a component architecture, we can now define the useful notions of path, trace, etc.

Definition 6. Given the model $M=\left\langle\mathcal{C}, \mathcal{R}_{\text {run }}, \rightarrow\right\rangle$, an evolution path (or a path for short) $\sigma$ of $M$ is a (possibly infinite) sequence of configurations $c_{0}, c_{1}, c_{2}, \ldots$ such that $\left.\left.\forall i \geq 0 . \exists r_{i} \in \mathcal{R}_{\text {run }} . c_{i} \stackrel{r_{i}}{\rightarrow} c_{i+1} \in \rightarrow\right)\right)$.

$W e$ use $\sigma(i)$ to denote the $i$-th configuration of a path $\sigma$. The notation $\sigma_{i}$ denotes the suffix path $\sigma(i), \sigma(i+1), \ldots$, and $\sigma_{i}^{j}$ denotes the segment path $\sigma(i), \sigma(i+1), \sigma(i+2), \ldots, \sigma(j-1), \sigma(j)$. The segment path is infinite in length when the last state of the segment is repeated infinitely often.

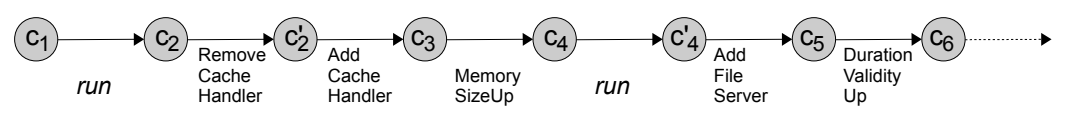

Fig. 3. Part of an evolution path of the HTTP server example

Example 2. A possible evolution path of the HTTP server is given in Fig. 3. In this path,

$-c_{1}$ is a configuration of the HTTP server without the CacheHandler and FileServer 2 components;

$-c_{2}$ is obtained from $c_{1}$ : the load value was changed following the running of the RequestHandler component;

$-c_{2}^{\prime}$ is the same configuration as $c_{2}$. Without the CacheHandler component, the RemoveCacheHandler reconfiguration cannot terminate, it is then rollbacked without any modification;

$-c_{3}$ is obtained from the configuration $c_{2}$ by adding CacheHandler, following the AddCacheHandler reconfiguration operation;

$-c_{4}$ is the configuration $c_{3}$ in which the memorySize value was increased;

$-c_{4}^{\prime}$ is the same configuration as $c_{4}$. The result of the running is not observable;

$-c_{5}$ is obtained from $c_{4}$ by adding the FileServer 2 component;

$-c_{6}$ is like the configuration $c_{5}$ but the durationValidity value was increased. 


\section{Temporal Logic for Dynamic Reconfigurations}

This section presents the syntax and the semantics of the temporal logic for dynamic reconfigurations. This logic, called FTPL, is inspired by the temporal logic in [21] designed to help Java programmers in writing formal specifications.

\subsection{Syntax of the Logic}

Let us first give the FTPL syntax:

\begin{tabular}{|c|c|c|}
\hline$<$ TempProp $>$ & $::=$ & $\begin{array}{l}\text { after }<\text { Events }><\text { TempProp }> \\
\text { before }<\text { Events }><\text { TraceProp }> \\
<\text { TraceProp }>\text { until }<\text { Events }> \\
<\text { TraceProp }>\text { unless }<\text { Events }> \\
\text { between }<\text { Events }><\text { Events }><\text { TraceProp }> \\
<\text { TraceProp }>\end{array}$ \\
\hline$<$ TraceProp $>$ & & $\begin{array}{l}\text { always ConfigProp } \\
\text { eventually ConfigProp } \\
\text { never ConfigProp } \\
<\text { TraceProp }>\wedge<\text { TraceProp }> \\
<\text { TraceProp }>\vee<\text { TraceProp }>\end{array}$ \\
\hline$<$ Events $>$ & $::=$ & $\begin{array}{l}<\text { Event }>,<\text { Events }> \\
<\text { Event }>\end{array}$ \\
\hline$<$ Event $>$ & $::=$ & $\begin{array}{l}\text { ReconfigOp called } \\
\text { ReconfigOp normal } \\
\text { ReconfigOp exceptional } \\
\text { ReconfigOp terminates }\end{array}$ \\
\hline
\end{tabular}

This language consists of different layers:

- the configurations properties,

- the reconfiguration operations,

- the trace properties,

- the temporal properties.

\subsection{Semantics of FTPL}

Let us now give the FTPL semantics. It is defined by induction on the form of the formulas.

Configuration properties Basically, there is a need to express properties on the configurations, i.e constraints on the architectural elements and the relations between them. These constraints are specified using first order logic formulas, sets and relational operations on the primitive sets and relations given in Sect. 3 .

Given the model $M$, we say that a configuration property $c p$ is valid on a configuration $c=\langle$ Elem, Rel $\rangle$, written $M, c \models c p$, when the evaluation of $c p$ on the configuration $c=\langle$ Elem, Rel $\rangle$ is true. When $M$ is understood, we simply write $c \models c p$. 
The configuration properties are expressed at different specification levels. At the component model level, the constraints are common to all the component architectures. In addition, some constraints must be expressed to restrict a family of component architectures (a profile level), or to restrict a specific component architecture (an application level).

Example 3. Let CacheConnected be a configuration property defined by

$\exists$ cache, getCache $\in$ Inter face. $($ ProvidedBy $($ cache $)=$ CacheHandler $\wedge$

RequiredBy (getCache $)=$ RequestHandler $\wedge$ Binding (cache $)=$ getCache $)$.

It expresses that the component CacheHandler is connected to RequestHandler. For the evolution path from Fig. 3 we have: $c 3 \models$ CacheConnected whereas $c 2 \not \models$ CacheConnected.

Event properties We want to observe reconfiguration action effects, for example when a reconfiguration is called or when it terminates, to specify and verify properties over them. Given a reconfiguration operation $r$ in $\mathcal{R}$, we consider the following events:

- $r$ called denoting that the reconfiguration $r$ has been invoked,

- $r$ normal denoting that the reconfiguration $r$ has terminated normally,

- $r$ exceptional denoting that the configuration $r$ has rollbacked.

Definition 7. Let $\sigma$ be an evolution path in $M$, and $r$ a reconfiguration operation in $\mathcal{R}$. Given an event property e, depicted $<$ Event $>$ in the FTPL syntax, its validity on the $i$-th configuration of $\sigma$, denoted $\sigma(i) \models e$, is inductively defined on the form of $e$ by:

$\begin{array}{|ll|}\sigma(i) \models r \text { called } & \text { iff } \exists \sigma(i+1) \cdot(\sigma(i) \stackrel{r}{\rightarrow} \sigma(i+1) \in \rightarrow) \\ \sigma(i) \models r \text { normal } & \text { iff } i>0 \wedge \sigma(i-1) \models r \text { called } \wedge \sigma(i-1) \neq \sigma(i) \\ \sigma(i) \models r \text { exceptional } & \text { iff } i>0 \wedge \sigma(i-1) \models r \text { called } \wedge \sigma(i-1)=\sigma(i) \\ \sigma(i) \models r \text { terminates } & \text { iff } \sigma(i) \models r \text { normal } \vee \sigma(i) \models r \text { exceptional }\end{array}$

Given an evolution path $\sigma$, and a list of event properties $E=e_{1}, \ldots, e_{n}$, depicted $<$ Events $>$ in the syntax, we say that $E$ is valid on the $i$-th configuration of $\sigma$, denoted $\sigma(i) \models E$, iff at least one event of the list $E$ is valid on $\sigma(i)$.

Example 4. Let us consider again the evolution path displayed in Fig. 3. We have: $c 3 \models$ MemorySizeUp called, $c 5 \models$ AddFileServer normal and $c 2 \models$ RemoveCacheHandler exceptional.

Trace Properties A trace property expresses a constraint which must be true when the component-based architecture changes, i.e on the evolution path.

Definition 8. Let $\sigma$ be an evolution path, and cp a configuration property. Given a trace property trp, depicted <TraceProp $>$ in the FTPL syntax, its validity on $\sigma$, denoted $\sigma=$ trp, is inductively defined on the form of trp by:

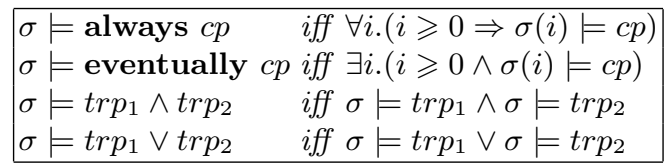


Intuitively,

- always $c p$ is valid on an evolution path $\sigma$ iff $c p$ is valid on each configuration of $\sigma$;

- eventually $c p$ is valid on an evolution path $\sigma$ iff $c p$ is valid on one configuration of $\sigma$, at least;

- the semantics of conjunction and disjunction is classical.

In the syntax a keyword never is introduced: never $c p$ being an abbreviation for always $\neg c p$.

Let us remark that architectural invariants as presented in $[16,18]$, can be handled within the FTPL framework by using always $c p$, where $c p$ represents the considered architectural invariant.

Temporal Properties Temporal properties are based on all the properties above, i.e. they exploit architectural constraints, event properties and trace properties, together with some temporal patterns, like in [21]. Let us recall that the SanTos Specification Pattern Project [13] has identified these temporal patterns as useful in practice.

Definition 9. Let $\sigma$ be an evolution path, E, $E_{1}$ and $E_{2}$ be lists of events, trp a trace property. Given a temporal property tpp, depicted $\langle$ TempProp $>$ in the FTPL syntax, its validity on $\sigma$, denoted $\sigma \models t p p$, is inductively defined on the form of tpp by:

$$
\begin{aligned}
& \sigma \models \operatorname{after} E \text { tpp } \quad \text { iff } \forall i .\left(i \geqslant 0 \wedge \sigma(i) \models E \Rightarrow \sigma_{i} \models t p p\right) \\
& \sigma \models \text { before } E \text { trp iff } \forall i .\left(i>0 \wedge \sigma(i) \models E \Rightarrow \sigma_{0}^{i-1} \models \operatorname{trp}\right) \\
& \sigma=\operatorname{trp} \text { until } E \quad \text { iff } \exists i .\left(i>0 \wedge \sigma(i) \models E \wedge \sigma_{0}^{i-1} \mid=\operatorname{trp}\right) \\
& \sigma \models \operatorname{trp} \text { unless } E \text { iff } \forall i .(i \geqslant 0 \wedge \sigma(i) \not \forall E \Rightarrow \sigma \mid \operatorname{trp}) \\
& \vee \exists i .\left(i \geqslant 0 \wedge \sigma(i) \models E \wedge \sigma_{0}^{i-1} \mid=\operatorname{trp}\right)
\end{aligned}
$$

Intuitively,

- the property after $E$ tpp is valid on an evolution path $\sigma$ iff the validity of the event property $E$ on a configuration of $\sigma$ implies the validity of the temporal property tpp on the suffix of $\sigma$ starting at this configuration;

- before $E \operatorname{trp}$ is valid on an evolution path $\sigma$ iff for each configuration of $\sigma$, the validity of $E$ on it means that the trace property trp is valid on the prefix of $\sigma$ before the considered configuration;

- trp until $E$ is valid on an evolution path $\sigma$ iff there is a configuration of $\sigma$ satisfying the event property $E$, and the trace property trp is valid on the prefix of $\sigma$ ending before this event occurs;

- trp unless $E$ is valid on an evolution path $\sigma$ iff either the event property $E$ is not valid on $\sigma$ implying that the trace property $\operatorname{tr} p$ is valid on $\sigma$, or there is a configuration of $\sigma$ satisfying the event property $E$, and the trace property trp is valid on the prefix of $\sigma$ before the corresponding event occurs;

A formula between $E_{1} E_{2} \operatorname{trp}$ has the same semantics as after $E_{1}$ (trp until $E_{2}$ ), i.e. the trace property $\operatorname{trp}$ is valid on the segment of $\sigma$ consisting of the configurations in-between the configuration where $E_{1}$ holds (including it), and the configuration where $E_{2}$ holds (excluding it). 


\subsection{Application to the HTTP Server Example}

Let us now illustrate the FTPL language use by expressing some properties for the example of HTTP server from Sect. 2.

et us consider a temporal property saying that after the invocation of the reconfiguration operation AddCacheHandler, the CacheHandler component is always connected to RequestHandler, i.e. the CacheConnected configuration property from Example 3 holds on the path configurations after the invocation. This property is valid on the evolution path $\sigma$ depicted in Fig. 3:

$$
\sigma \models \text { after AddCacheHandler called always CacheConnected. }
$$

The following property expresses an architectural constraint saying that at least there is always one file server component connected.

$$
\begin{gathered}
\text { always ( } \exists \text { getServer } \in \text { Interface. }(\text { RequiredBy }(\text { getServer })= \\
\text { RequestDispatcher } \wedge \exists i \in \text { Interface.Binding }(i)=\text { getServer }))
\end{gathered}
$$

Let us now specify that the deviation must always be lower than 50 until the AddCacheHandler reconfiguration operation terminates normally:

$$
\begin{gathered}
\text { (always } \\
\exists \text { deviation } \in \text { Parameter. }(\text { ParameterOf }(\text { deviation })=\text { RequestHandler } \\
\wedge \text { deviation }<50)) \text { until AddCacheHandler normal }
\end{gathered}
$$

The following property says that between the exceptional termination of either the MemorySizeUp reconfiguration or the DurationValidityUp reconfiguration, and the normal termination of the AddCacheHandler reconfiguration operation, the number of used file servers is greater than 1:

$$
\begin{gathered}
\text { between (MemorySizeUp exceptional, DurationValidityUp exceptional) } \\
\text { (addCacheHandler normal) } \\
(\exists \text { getServer } \in \text { Interface. }(\text { RequiredBy }(\text { getServer })=\text { RequestDispatcher } \wedge \\
\left.\exists i, i^{\prime} \in \text { Interface. }\left(i \neq i^{\prime} \wedge \text { Binding }(i)=\operatorname{getServer~} \wedge \text { Binding }\left(i^{\prime}\right)=\text { getServer }\right)\right)
\end{gathered}
$$

These examples show that architectural invariants and properties on immediate predecessors or target configurations of reconfiguration operations can be expressed by FTPL formulas. Further, they show that FTPL is more expressive than the proposals in [12]. Indeed, FTPL allows expressing event properties and temporal properties involving different kinds of properties satisfying temporal patterns which have been shown useful for practical applications.

\subsection{On the Expressiveness of FTPL}

Before considering FTPL temporal properties, let us recall that configuration properties are first order logic formulas.

Let us now consider temporal patterns. As explained in Sect.4, FTPL has been inspired by proposals in [13], and works on a temporal extension of JML [21, $9,14]$, called JTPL. The semantics of JTPL temporal formulas and translation 
rules into JML annotations are detailed in [21] for safety properties and in [9] for liveness properties.

Let $\operatorname{LTL}_{k}()$ denote a function translating the FTPL properties of the kind $k$ into LTL properties. We adapt the above-mentioned works and propose the following translation of FTPL patterns into LTL formulas. In this translation $c p$ is a configuration property, $t r p, t r p_{1}$ and $t r p_{2}$ are trace properties, $E, E_{1}$ and $E_{2}$ are lists of event properties, and tpp is a temporal property. In FTPL, there is a way to decide whether a list of event properties is valid on a configuration or not. The following functions suppose that the same decision procedure exists in LTL.

Leaving aside the FTPL and LTL models, it is easy to see that FTPL trace properties can be rewritten in LTL as follows:

\begin{tabular}{|c|c|}
\hline $\operatorname{LTL}_{\text {Trace }}($ always $c p)$ & $\mathrm{G}(c p)$ \\
\hline LTL $_{\text {Trace }}($ eventually $c p)$ & $\mathrm{F}(c p)$ \\
\hline $\operatorname{LTL}_{\text {Trace }}\left(\operatorname{trp}_{1} \& \operatorname{trp}_{2}\right)$ & $\mathrm{LTL}_{\text {Trace }}\left(\operatorname{trp}_{1}\right) \wedge \mathrm{LTL}_{\text {Trace }}\left(\operatorname{trp}_{2}\right)$ \\
\hline $\mathrm{LTL}_{\text {Trace }}\left(\operatorname{tr} p_{1} \mid \operatorname{tr} p_{2}\right)$ & $\mathrm{LTL}_{\text {Trace }}\left(\operatorname{trp}_{1}\right) \vee \mathrm{LTL}_{\text {Trace }}\left(\operatorname{trp}_{2}\right)$ \\
\hline
\end{tabular}

For example, if in LTL atomic properties could be configuration properties, the safety property specifying that always there is at least one file server component connected, would be written in LTL as follows:

$\mathrm{G}(\exists$ getServer $\in$ Inter face. (RequiredBy (getServer) $=$ RequestDispatcher $\wedge \exists i \in$ Interface.Binding $(i)=$ getServer $))$

For temporal properties, we have:

\begin{tabular}{|c|c|}
\hline $\operatorname{LTL}_{T e m p}($ after $E t p p)$ & $\mathrm{G}\left(E \Rightarrow \mathrm{LTL}_{T e m p}(t p p)\right)$ \\
\hline $\operatorname{LTL}_{T e m p}($ after $E \operatorname{trp})$ & $\mathrm{G}\left(E \Rightarrow \mathrm{LTL}_{\text {Trace }}(\operatorname{tr} p)\right)$ \\
\hline LTL $_{T e m p}($ before $E \operatorname{trp})$ & $\mathrm{F}(E) \Rightarrow \mathrm{LTL}_{\text {Trace }_{B}}(E, \operatorname{trp})$ \\
\hline $\operatorname{LTL}_{T e m p}(\operatorname{trp}$ until $E)$ & $\mathrm{F}(E) \wedge \operatorname{LTL}_{\text {Trace }_{B}}(E, \operatorname{trp})$ \\
\hline $\operatorname{LTL}_{T e m p}(t r p$ unless $E)$ & $\operatorname{LTL}_{\text {Trace }_{C}}(E, \operatorname{trp})$ \\
\hline $\operatorname{LTL}_{T e m p}\left(\right.$ between $\left.E_{1} E_{2} t r p\right)$ & $\operatorname{LTL}_{T e m p}\left(\right.$ after $E_{1}\left(\operatorname{trp} p\right.$ until $\left.\left.E_{2}\right)\right)$ \\
\hline $\mathrm{LTL}_{T e m p}(\operatorname{trp})$ & $\mathrm{LTL}_{\text {Trace }}(\operatorname{trp})$ \\
\hline
\end{tabular}

where:

\begin{tabular}{|l|l|}
\hline $\operatorname{LTL}_{\text {Trace }_{B}}(E$, always $c p)$ & $c p \cup E$ \\
\hline $\operatorname{LTL}_{\text {rrace }_{B}}(E$, eventually $c p)$ & $\neg(\neg c p \cup E)$ \\
\hline $\operatorname{LTL}_{\text {race }_{C}}(E$, always $c p)$ & $\mathrm{G}(c p) \vee(c p \cup E)$ \\
\hline $\operatorname{LTL}_{\text {Trace }_{C}}(E$, eventually $c p)$ & $\mathrm{F}(c p) \wedge \neg(\neg P \cup E)$ \\
\hline
\end{tabular}

Remark that a trace property is translated into LTL according to the temporal context in which the property is used, that is why we define two auxiliary functions $\mathrm{LTL}_{\text {Trace }_{B}}$ and $\mathrm{LTL}_{\text {Trace }_{C}}$. The $\mathrm{LTL}_{\text {Trace }}$ function translates a trace property which either does not depend on a temporal property, or is inside an after temporal property. The $\mathrm{LTL}_{\text {Trace }_{B}}$ function is used to translate a trace property which is inside a before or an until temporal properties. Finally, the LTL $_{\text {TraceC }_{C}}$ function translates a trace property bounded by a unless temporal property. 
For example, the property specifying that the deviation must always be lower than 50 until the AddCacheHandler reconfiguration operation terminates normally can be written in LTL as follows:

$$
\begin{aligned}
& \mathrm{F}(\text { AddCacheHandler normal }) \wedge( \\
& \exists \text { deviation } \in \text { Parameter. }(\text { ParameterOf }(\text { deviation })=\text { RequestHandler } \\
&\wedge \text { deviation }<50) \cup \text { AddCacheHandler normal })
\end{aligned}
$$

\section{Application to the Fractal Component Model}

The Fractal component model [10] is one of the motivations of the present work because of its native support for dynamic architectures. Fractal also provides means for introspection and reconfigurations. Existing implementations for Fractal and its extensions offer a framework to experiment with FTPL-based reconfigurations. This section briefly describes some Fractal features and the existing language support for reconfigurations, before reporting on our experiments.

\subsection{Overview of Fractal, FPath and FScript}

The Fractal model is a hierarchical and reflective component model intended to implement, deploy and manage software systems [10]. A Fractal component is both a design and a run-time entity that consists of a unit of encapsulation, composition and configuration. A component is wrapped in a membrane which can show and control a casually connected representation of its encapsulated content. This content is either directly an implementation in case of a primitive component, or sub-components for composite components.

FPath [12] is a domain-specific language inspired by the XPath language that provides a notation and introspection mechanisms to navigate inside Fractal architectures. FPath expressions use the properties of components (e.g. the value of a component attribute or the state of a component) or architectural relations between components (e.g. the subcomponents of a composite component) to express queries about Fractal architectures.

FScript [12] is a language that allows the definition of reconfigurations of Fractal architectures. FScript integrates FPath seamlessly in its syntax, FPath queries being used to select the elements to reconfigure. To ensure the reliability of its reconfigurations, FScript considers them as transactions and integrates a back-end that implements this semantics on top of the Fractal model.

\subsection{From the FTPL Model to Fractal}

As explained above, the architectural model presented in Sec. 3 has been developed to capture the Fractal component model, among other component-based models with reconfigurations, like CCM, GCM, etc. To illustrate our proposals, in this section we give a part of the Http Server example encoded using our model (Fig. 4) as well as its implementation in FractalADL (Fig. 5). 


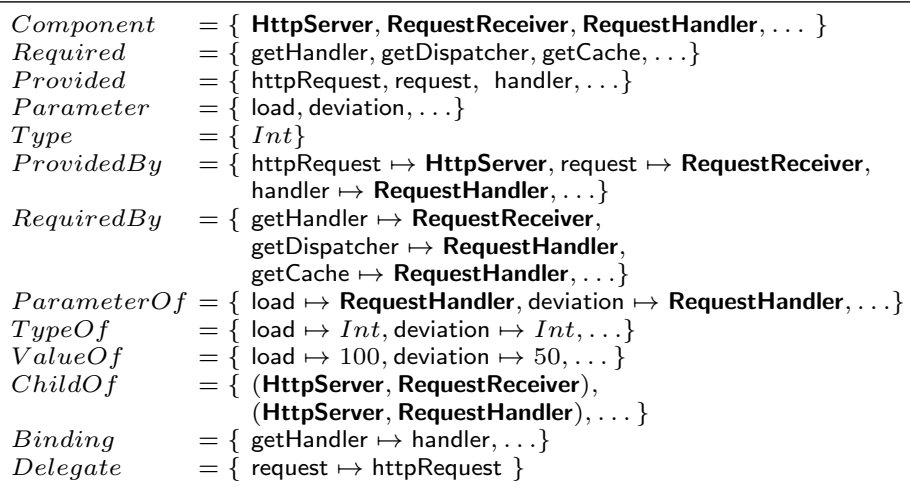

Fig. 4. HttpServer example using Definitions 2 and 3

Let us recall that FractalADL ${ }^{4}$ is the architecture description language for Fractal which allows implementing the Fractal component model.

We then use the FScript language to specify the reconfiguration operations presented in Sec. 3, and the FScript tool support to execute them. FScript is focused on the manipulation of architectural concepts and provides complete control of the architecture of the systems modeled in Fractal. Concretely, FScript takes an architecture of a current Fractal configuration and dynamically changes it according to a FScript file in order to create a new target architecture. The FScript implementation features guarantee that FScript reconfigurations always terminate and keep the system in a consistent and usable state.

For example, we specify the AddCacheHandler reconfiguration in FScript as presented in Fig. 6. This reconfiguration consists in creating a new instance of CacheHandler (name) and in specifying its name (set-name). Then, the component is integrated into the architecture (add) and the binding with the component RequestHandler is set (bind). Finally, the component CacheHandler is started (start).

\subsection{Dynamic Verification}

We now report on our experiments evaluating the feasibility of a run-time monitoring of FTPL properties. The monitoring on the execution of the architectural reconfiguration model depends on the property to be verified; It is either the satisfiability of a configuration property on one configuration, or the satisfiability of a temporal property on a sequence of component-based system architectures.

Verification of configuration properties. We use the FPath language support to verify a configuration property on one configuration. Indeed, any first order

\footnotetext{
${ }^{4}$ http://fractal.ow2.org/fractaladl/
} 


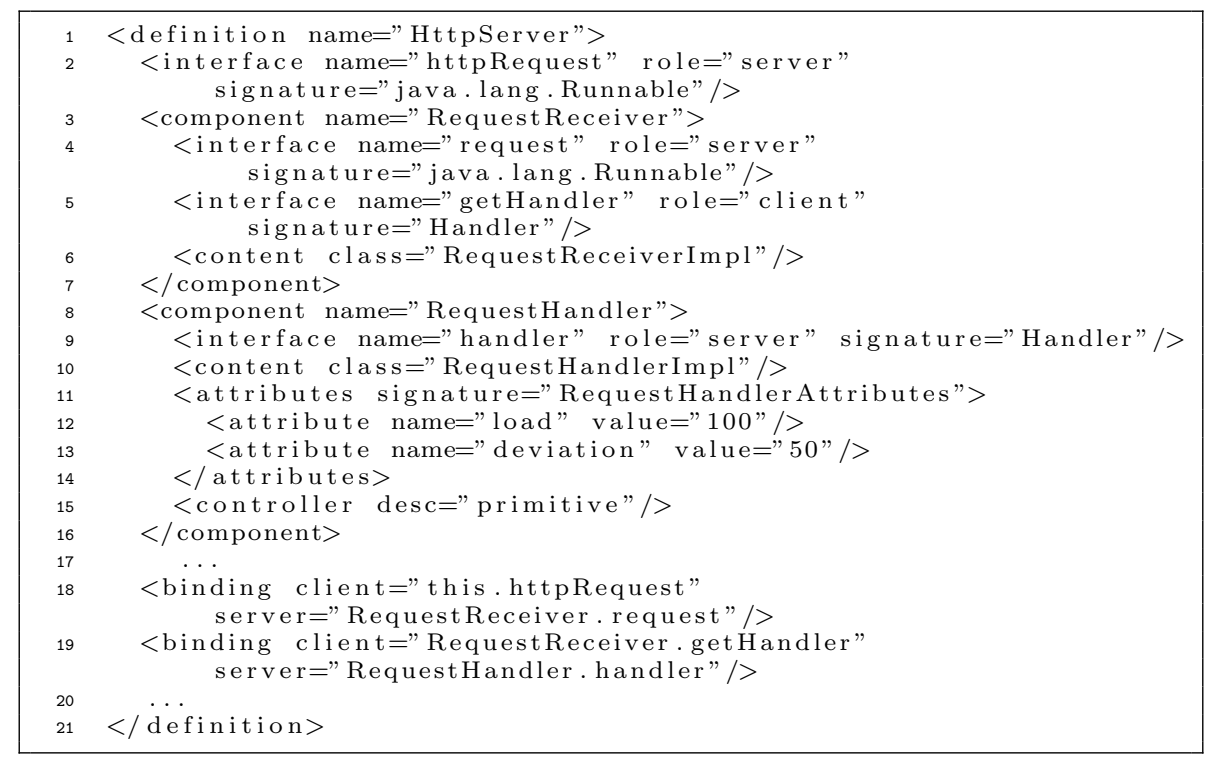

Fig. 5. HTTP Server example in FractalADL

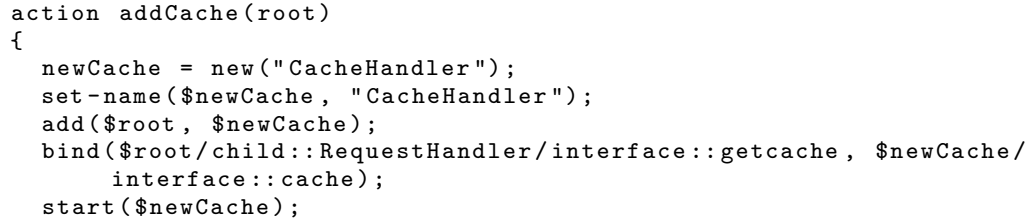

Fig. 6. AddCacheHandler Reconfiguration specified in FScript

logic formula specifying a configuration property can be translated into an FPath expression, the FPath language having the same expressive power [16].

For example, the CacheConnected configuration property from Example 3 can be expressed in FPath by:

\$HttpServer/child: : RequestHandler/interface: : getCache/binding : : cache/ $\hookrightarrow$ component: : CacheHandler

Verification of temporal properties. Once the configuration properties are handled thanks to FPath, there is a need to deal with FTPL temporal properties. In [7], it is shown that the monitoring works well on specific safety properties. In FTPL the safety properties are properties containing only the keywords after, before, unless and always; The safety properties are also properties containing the eventually keyword iff they contain the before keyword to bound the eventually part. The other properties are liveness properties. 
We have studied the feasibility of the safety properties monitoring by developing a controller in Fractal. This controller supervises the properties of interest each time a reconfiguration operation occurs. It retains the configurations appearing during the system execution to build a history and to use it for verification purpose. In order to make the monitoring easier, the controller divides the property into sub-properties and keeps each sub-property until it manages to validate it. For example, for the property after AddCacheHandler called always CacheConnected, the controller tries first to find a configuration where the AddCacheHandler called event property holds. Once such a configuration is found, the controller continues with the monitoring of the CacheConnected configuration property for all the following configurations.

The FTPL properties can be divided into two classes: the properties dealing with the past and the properties dealing with the future. As it is possible to determine what has happened in the past thanks to the history built by the controller, all the past properties can be monitored. But, due to the run-time verification, there are properties that cannot be ensured by the controller. For any property about the future containing always, the controller only ensures that the current configuration does not violate the property at the moment. For any property about the future containing eventually key-word, the controller cannot conclude neither.

As explained before, the controller is able to monitor the safety properties about the past, where as for the properties about the future, it only ensures their non-violation until the current configuration. An LTL-derived logic is proposed in [8] to specify and verify that a system behaviour will "presumably" conform or violate the property in the future. In [7], the monitoring of safety properties necessitates time intervals bounded in the past as well as in the future. To go further within the Fractal-based approach, and to handle liveness properties, a solution would be to exploit a variant specification of the Loop clause [14], to which liveness properties can be reduced.

\section{Conclusion}

In this paper we have developed a theoretical framework for dynamic reconfigurations of component-based systems. As a calculus for expressing and analysing reconfiguration and integrity constraints, we have utilised linear temporal logic, since formulas are interpreted over configuration sequences which naturally represent dynamic behaviour of component-based systems. For the Fractal component model, we have studied the feasibility of monitoring dynamic reconfigurations during system lifetime.

Related work Dynamic reconfiguration of distributed applications is an active research topic $[1,2,7,18]$ motivated by practical applications like those modelled in Fractal [10]. In the context of dynamic reconfigurations, ArchJava [3] gives 
means to reconfigure Java architectures, and the ArchJava language guarantees communication integrity at run-time. Barringer and al. also give a formal temporal logic based framework to reason about the evolution of systems [5].

The idea of using graph-based models to specify dynamic reconfigurations is not new at all. In [4], a temporal logic is proposed to specify and verify properties on graph transformation systems. In the Fractal-based framework, in $[17,18]$ the authors have defined integrity constraints on a graph-based representation of Fractal, to specify the reliability of component-based systems. There are tools to allow the user to ensure the reliability of those reconfigurations at run-time.

Our model in Sect. 3 is closely related to the model proposed in [18] for the Fractal component systems but unlike [18], our model lays down only general architectural constraints. In this sense it can be considered as a generalisation of the Fractal-oriented model. Moreover, our model seems to be general enough to give operational semantics to other component-based systems. On the integrity and architectural constraint side, the FTPL logic allows us to specify architectural constraints more complex that only architectural invariants in [12].

Among other applications, our proposals aims at an active monitoring of component-based systems. The active monitoring involves interpreting a configuration data set and acting on those data to (re-)configure the system accordingly. This may simply be a validation of the target configuration, or a reconfiguration operation interruption. In $[7,6]$, Basin and al. have shown the feasibility of monitoring temporal (safety) properties and, more recently, security properties using a runtime monitoring approach for metric First-order temporal logic (MFOTL). The semantics of MFOTL has been defined with respect to timed temporal structures. Like the model in [7], our model is a first-order structure, but instead of considering a sequence of time stamps, we focus on reconfiguration operations. Although our main motivation and hence the model are different, their algorithms for monitoring temporal safety properties would be adapted for performing dynamic reconfigurations of component-based systems.

\section{References}

1. M. Aguilar Cornejo, H. Garavel, R. Mateescu, and N. De Palma. Specification and Verification of a Dynamic Reconfiguration Protocol for Agent-Based Applications. Research Report RR-4222, INRIA, 2001.

2. N. Aguirre and T. Maibaum. A temporal logic approach to the specification of reconfigurable component-based systems. Automated Software Engineering, 2002.

3. J. Aldric. Using types to enforce architectural structure. In In WICSA'08, February 2008, pages 23-34, 2008.

4. P. Baldan, A. Corradini, B. König, and A. Lluch Lafuente. A temporal graph logic for verification of graph transformation systems. In WADT'06: Proceedings of the 18th international conference on Recent trends in algebraic development techniques, pages 1-20. Springer-Verlag, 2007.

5. H. Barringer, D. M. Gabbay, and D. E. Rydeheard. From runtime verification to evolvable systems. In $R V$, volume 4839 of $L N C S$, pages 97-110. Springer, 2007.

6. D. A. Basin, F. Klaedtke, and S. Müller. Policy monitoring in first-order temporal logic. In CAV 2010, UK, July, 2010, volume 6174 of $L N C S$, pages 1-18, 2010. 
7. D. A. Basin, F. Klaedtke, S. Müller, and B. Pfitzmann. Runtime monitoring of metric first-order temporal properties. In IARCS, FSTTCS 2008, India, volume 2 of LIPIcs, pages 49-60. Schloss Dagstuhl - Leibniz-Zentrum fuer Informatik, 2008.

8. A. Bauer, M. Leucker, and C. Schallhart. Comparing LTL Semantics for Runtime Verification. Journal of Logic and Computation (JLC), 2010.

9. F. Bellegarde, J. Groslambert, M. Huisman, J. Julliand, and O. Kouchnarenko. Verification of liveness properties with JML. Technical report RR-5331, INRIA, 2004.

10. E. Bruneton, T. Coupaye, M. Leclercq, V. Quéma, and J.-B. Stefani. The fractal component model and its support in java. Softw., Pract. Exper., 36(11-12):12571284, 2006.

11. F. Chauvel, O. Barais, N. Plouzeau, I. Borne, and J.-M. Jézéquel. Composition et expression qualitative de politiques d'adaptation pour les composants Fractal. In GDR GPL 2009, Toulouse, France, January 2009.

12. P.-C. David, Th. Ledoux, M. Léger, and Th. Coupaye. FPath and FScript: Language support for navigation and reliable reconfiguration of Fractal architectures. Annales des Télécommunications, 64(1-2):45-63, 2009.

13. M. B. Dwyer, G. S. Avrunin, and J. C. Corbett. Patterns in property specifications for finite-state verification. In ICSE, pages 411-420, 1999.

14. A. Giorgetti, J. Groslambert, J. Julliand, and O. Kouchnarenko. Verification of class liveness properties with java modelling language. IET Software, 2008.

15. J. O. Kephart and D. M. Chess. The vision of autonomic computing. Computer, 36(1):41-50, 2003.

16. M. Léger. Fiabilité des Reconfigurations Dynamiques dans les Architectures à Composant. PhD thesis, Ecole Nationale Supérieure des Mines de Paris, 2009.

17. M. Léger, Th. Ledoux, and Th. Coupaye. Reliable dynamic reconfigurations in the fractal component model. In ARM'0\%, pages 1-6. ACM, 2007.

18. M. Léger, Th. Ledoux, and Th. Coupaye. Reliable dynamic reconfigurations in a reflective component model. In CBSE 2010, volume 6092 of $L N C S$, pages 74-92. Springer-Verlag, 2010.

19. Z. Manna and A. Pnueli. The Temporal Logic of Reactive and Concurrent Systems: Specification. Springer-Verlag, 1992.

20. B. Redmond and V. Cahill. Supporting unanticipated dynamic adaptation of application behaviour. In ECOOP 2002, Malaga, Spain, June 10-14, 2002, volume 2374 of Lecture Notes in Computer Science, pages 205-230. Springer, 2002.

21. K. Trentelman and M. Huisman. Extending jml specifications with temporal logic. In AMAST 2002, volume 2422 of LNCS, pages 334-348, 2002. 